nizations (the World Health Organization (WHO), the United Nations Children's Fund (UNICEF), etc.).

During the given period, medical assistance was given to $>150,000$ patients, including 38,000 children. More than 1,300 patients with active TB were detected, and were given in-patient treatment, and $>3,500$ contact persons received pharmacotherapy. In-patient assistance was provided to $>18,000$ patients in the field hospitals, $>4,000$ people in the hospitals situated near the boundaries of the Republic, and $>700$ people received highly technological types of assistance outside the Republic. More than 15,000 children were vaccinated. Outbreaks of infectious diseases and group diseases among the temporarily displaced persons from the Chechen Republic were not observed during that period.

A proposed system for providing medical assistance to the temporarily displaced populations in Northern Caucasus showed high efficiency and may be recommended for use in other countries in analogous situations.

Keywords: assistance; efficiency; Chechen Republic; health support; Northern Caucasus; vaccinations

Prebosp Disast Med 2005;20(2):s76-\$77

\section{Provision of Medical Support in Large-Scale Disasters: Level of Commitment by the Municipal Bodies in Prefectures and Government Ordinance-Designated Cities in Japan$$
\text { U. Hiroko, }{ }^{1} \text { A. Shigeaki, }{ }^{1} \text {. S. Yasuyuki, }{ }^{1} \text { Y. Takao }{ }^{2}
$$ \\ T. Koicbi, ${ }^{2}$ K. Kenji $i^{1}$ \\ 1. Department of Pharmaceutical Services, Hiroshima Uni, Japan \\ 2. Emergency and Critical Care Medicine, Hiroshima Uni, Japan}

Objective: When a disaster occurs, the municipal bodies must perform prompt and effective allocation of comprehensive disaster medical support. However, establishing the appropriate system for disaster medical support is in its infancy in most of the municipal bodies in Japan. In this study, the current issues affecting the municipal bodies in Japan were surveyed and analyzed in regards to the system for provision of disaster medical support, and the results were analyzed in order to establish future recommendations. Methods: In October 2004, a questionnaire was sent to a total of 60 Japanese governmental bodies and the responses were collected.

Results: Of the 60 bodies, $37(68 \%)$ completed and returned the questionnaire. Nine (22\%) have a special working committee for the provision of disaster medical support. In regards to developing operational guidelines for disaster medical support management, 25 (68\%) of the respondents have designed their own guidelines, while 34 (92\%) have stockpiled medical kits and supplies for a disaster in their storage bases. However, of the $68 \%$ who designed guidelines, $22 \%$ actually made the availability of the guidelines known publicly; of the $92 \%$ with stockpiled supplies, $19 \%$ made the existence of the stockpiles publicly known. In regards to the system for processing information at the time of a disaster, $38 \%$ of the respondents have set up a system that enables them to collect information from all the relevant organizations including local municipal offices, major hospitals, and related representatives. This study did not find any municipal body that regularly organizes workshops or training sessions in order to train future managers or coordinators for overseeing efficient provision of disaster medical support.

Discussion: The results of the survey showed that: (1) no practical working relationships have been established among related representatives to deal with disaster medical support at the time of a disaster; and (2) a number of municipal bodies have designed guidelines for managing disaster medical support, have arranged to have medical kits and supplies for disaster in stock, and have set up a system to deal with a flood of information in a disaster. However, few municipal bodies have exercised an adequate level of public relations or organized official training sessions in their municipal areas. Thus, it is feared that the aforementioned publicity and facilities may be in vain at the time of a disaster, as they would not function in the optimal manners intended. Each municipal body should take immediate action in the following areas: (1) exercising appropriate professional relations with the medical organizations/institutions and with the public; (2) developing and implementing a system for disaster medical support through proper establishment of working relations among relevant representatives; and (3) offering educational programs to the members of the healthcare community in order to increase the awareness of disaster medical support needs.

Keywords: guidelines; Japan; large-scale disaster; medical; municipal bodies; publicity; support

Prebosp Disast Med 2005;20(2):s77

\section{Lapses in Response to Road Traffic Crashes in Nigeria} E. Ebikbamenor; A. Ogbemudia

Save Accident Victims Association of Nigeria (SAVAN)/ University of Benin (UNIBEN), Nigeria

Introduction: The World Health Organization (WHO) statistics show that since the advent of the automobile, more than 32 million persons have died from traffic crashes, and an average of 700,000 persons die annually. Data from the WHO further reveal that the number of annual deaths due to traffic crashes is more than the number who have died from earthquakes, floods, typhoons and all other natural disasters combined. Another global report from the WHO reveals that developed countries or highly motorized countries (HMC) account for $85 \%$ of vehicle ownership, while transitional countries account for only $15 \%$ of car ownership. Paradoxically, developed countries account for only $15 \%$ of road traffic crashes while transitional countries account for $85 \%$ of road traffic crashes. Based on data from Federal Road Safety Commission (FRSC), Nigerian Police, and the Save Accident Victims Association of Nigeria (SAVAN) organization, incidents of road traffic crashes associated with morbidity and mortality are astronomically on the rise. Analysis of these data shows that from 1955-2004, deaths per crash illustrate poor coordination and several lapses of first responders, hence unacceptable deaths are recorded per-accident cases. Non-existing prehospital structures at all levels have contributed to unac- 
ceptably high incidences of mortality/morbidity from road traffic crashes (RTC).

Objectives: The objective of this study is to analyze Nigerian crash diaries from the pre-independence era to the present date, highlight various lapses involved to minimize RTCs on the highways, and appropriate protocols for team management of such victims.

Methods: Police, the FRSC, and all SAVAN-designated centers were utilized to collate data of crashes involving vehicles and Okada, and also recorded the number of injured persons with associated mortality as a result of the crash. Diurnal variations, vehicular distributions, and geographic locations of the crash were documented. The structure of a non-governmental organization, such as SAVAN, was used to determine the biological data of the RTC victims.

Results: In comparison with other countries, Nigeria recorded one death per $<3$ cases, compared to countries, like France, who recorded only one death in $>180$ cases. Poor coordination of first responders and other stakeholders enormously contributed to high mortality.

Conclusions: Nigeria needs a constant safety training program, persistent media advocacy, enactment/enforcement of appropriate legislation against all offenders of traffic regulations, and activation of all stakeholders, prior to, during, and after traffic accidents to save more lives on the highway. Keywords: Federal Road Safety Commission (FRSC); first responder lapses; Nigeria; traffic crashes

Prehosp Disast Med 2005:20(2):s77-s78

\section{Prehospital Emergency Care in Severe Bus and Coach Crashes-Lessons Learned in Sweden \\ P. Albertsson \\ Umeã University, Sweden}

Buses and coaches rarely are involved in severe crashes, but when they are, the numbers of casualties are usually high due to the large number of occupants traveling in the vehicles. Coach crashes in which the vehicle rolls $90^{\circ}$ or more is a type of severe crash that exposes occupants to high risks of injury. If the post-crash position of the vehicle of a rollover is $90^{\circ}$ on its side, this imposes a great challenge for emergency care, rescue services, and medical teams. Injured and fatally trapped occupants might be located inside, outside, and underneath the crashed coach. A number of occupants with minor injuries also need to be attended. As a consequence, there are a number of crucial tasks to deal with in a short period of time, which in turn, emphasizes the importance of a proper organization based on education and training in order to facilitate the rescue work. Systematic training of rescue and ambulance teams in cooperation at a crash site may reduce the extrication time of entrapped victims in coach crashes.

Experiences from a number of severe coach crashes in Sweden were gathered. The main findings were that: (1) normal triage had to be set aside, in favor of evacuating the passengers, starting with those closest to the exit; (2) use of ordinary equipment is not always possible inside the crashed vehicle; (3) a $90^{\circ}$ position of a coach makes the working conditions difficult for the ambulance and fire personnel, with access only through the front and/or rear window and the roof hatches; (4) knowledge of reading kinematics is useful; (5) occupants may be still trapped and alive under the crashed coach; (6) in situations occurring a substantial distance from hospitals, a casualty clearing station is preferable to a more rapid procedure, such as a "load and go"; and (7) previous exercises where rescue team members have worked together is valuable in real situations.

Furthermore, the gathered experiences were the start for a cooperation project between the rescue and emergency service within the area "heavy rescue".

Keywords: buses; crashes; emergency care; rescue; Sweden; vehicles Prehosp Disast Med 2005;20(2):s78

\section{Role of Photography in the Management of Road Traffic Crashes in Nigeria}

N. Ehigie

Save Accident Victims Association of Nigeria (SAVAN), Nigeria

Most deaths, injuries, and economic losses in Nigeria are due to preventable car crashes. The World Health Organization (WHO) estimated the annual global cost of crashes at US $\$ 520$ billion in 1998. In developed countries, those at risk of injury and deaths are vehicle occupants, while those at risk in developing countries are pedestrians, cyclists, motorcyclists, and users of informal modes of public transportation. This study highlights the growing trend in road traffic crashes and the role of photography in examining this trend and its use in identification of dead or unconscious victims in six Save Accident Victims Association of Nigeria (SAVAN) hospitals. Photography helps to translate ideas into visuals, symbols, or ideograms. Due to the lack of available forensic medicine in developing countries, identifying unknown victims of major disasters or road traffic crashes can be difficult. Hence, the use of photography has become a major approach for establishing the identity of such victims.

Cultural and religious factors have not helped facilitate the identification process because some tribes and religions do not allow post-mortem examinations or other forensic procedures, making photographic documentation very vital to assisting professionals establish the identity of the victims. Its availability and cost-effectiveness makes it readily accessible to everyone in Nigeria. Identification can be cumbersome due to the mode used for rescue, corrupt rescue officers, lack of proper identity cards, and poor information and communication management.

Keywords: culture; management; Nigeria; photography; religion; Save Accident Victims Association of Nigeria (SAVAN); traffic crashes

Prebosp Disast Med 2005;20(2):s78

\section{Impaired Driving In Southern Nigeria Due To Alcohol}

E. Ehikhamenor

Save Accident Victims Association of Nigeria (SAVAN)/ University of Benin (UNIBEN), Nigeria

Introduction: Developed countries, also regarded as highly motorized countries (HMC), recently acknowledged a decline in drinking and driving, especially during the 1980s. Improved laws, enhanced enforcement, and public 